\title{
Unidad didáctica como estrategia pedagógica para el fortalecimiento de la lectura crítica en los estudiantes del grado undécimo de la institución educativa Alonso Carvajal Peralta ${ }^{1}$
}

\author{
Didactic unit as a pedagogical strategy for the \\ strengthening of critical reading in eleventh grade students of \\ the Alonso Carvajal Peralta educational institution \\ Unidade didática como estratégia pedagógica para o \\ fortalecimento da leitura crítica em alunos da décima \\ primeira série do Alonso Carvajal Peralta instituição \\ acadêmica
}

Juan Manuel Ochoa Gamboa

Candidato a magister en educación Universidad Autónoma de Bucaramanga jochoa378@unab.edu.co

\section{Resumen}

El trabajo de investigación surge de la necesidad de fortalecer la lectura crítica en los estudiantes del grado undécimo, ya que después de analizar los resultados de las pruebas Saber se identificó que los niveles de lectura de los estudiantes no eran los adecuados. Para ello, se consultaron diversas investigaciones concernientes a la problemática mencionada, que a su vez sirvieron de estado del arte para el proceso de investigación, siendo estos trabajos de grado realizados en maestrías y doctorados a nivel nacional e internacional respectivamente, vale la pena resaltar que después de realizar una búsqueda exhaustiva a nivel regional no se encontraron investigaciones que aportaran significativamente al proceso.

\footnotetext{
${ }^{1}$ El presente artículo esta elaborado con base al trabajo de grado "La Unidad Didáctica Como Estrategia Para Promover La Lectura Crítica En Los Estudiantes Del Grado Undécimo De La I.E. Alonso Carvajal Peralta, De Chitagá (N.S)" para obtener el título de Magister en Educación, con la Universidad Autónoma de Bucaramanga. UNAB, dentro del grupo de investigación: Educación y Lenguaje en la línea investigativa Prácticas Pedagógicas y dirigido por la Mg. Yolanda Villamizar de camperos.
} 
El principal objetivo planteado fue promover la lectura crítica en los estudiantes, partiendo de la base de determinar el nivel de lectura (literal, inferencial y crítico) de los estudiantes a través de una prueba diagnóstica. Posteriormente se diseñó e implementó una unidad didáctica como estrategia pedagógica innovadora, que por medio de sus actividades enfocadas en el aprendizaje significativo permitiera el fortalecimiento de la lectura crítica. Todo lo anterior, se enmarcó en la metodología de la investigación-acción con un enfoque cualitativo pues todo el proceso es autorreflexivo e indaga sobre las oportunidades de mejora tanto del docente como del estudiante; además se aplicó en un contexto pedagógico natural, arrojando datos descriptivos y dejando ver necesidades sociales, culturales, pedagógicas, didácticas, entre otras.

Seguidamente, se consiguió determinar el nivel de lectura de cada uno de los estudiantes y la unidad didáctica, elaborada como estrategia pedagógica, se ejecutó en su totalidad y fue de agrado para ellos. Por último, se valoró el avance de cada uno de los estudiantes en los tres niveles de lectura aplicando una prueba final.

Para finalizar, se pudo determinar que se debe fortalecer la lectura literal, con todo lo que esto implica, para que los demás niveles se desarrollen adecuadamente, teniendo en cuenta que dicho fortalecimiento debe estar enfocado también en la parte actitudinal y motivacional de los estudiantes.

Palabras claves: Niveles de lectura, lectura crítica, unidad didáctica, aprendizaje significativo.

\begin{abstract}
The research work arises from the need to strengthen the critical reading in the eleventh grade students, since after analyzing the results of the Saber tests it was identified that the reading levels of the students were not adequate. To do this, several inquiries were consulted concerning the aforementioned problems, which in turn served as a state of the art for the research process, with these master's and doctoral studies carried out at the national and international levels, respectively, it is worth noting that After conducting a comprehensive search at the regional level, no research was found that significantly contributed to the process.

The main objective was to promote critical reading in students, based on the basis of determining the level of reading (literal, inferential and critical) of students through a diagnostic test. Subsequently, a didactic unit was designed and implemented as an innovative pedagogical strategy, which, through its activities focused on meaningful learning, allowed the strengthening of critical reading. All of the above was framed in the methodology of action research with a qualitative approach because the
\end{abstract}


whole process is self-reflective and inquires about the improvement opportunities for both the teacher and the student; It was also applied in a natural pedagogical context, yielding descriptive data and revealing social, cultural, pedagogical and didactic needs, among others.

Afterwards, it was possible to determine the reading level of each one of the students and the didactic unit, developed as a pedagogical strategy, was executed in its entirety and was liked by them. Finally, the progress of each of the students in the three reading levels was assessed by applying a final test.

Finally, it could be determined that the literal reading should be strengthened, with all that this implies, so that the other levels develop properly, bearing in mind that this strengthening must also be focused on the attitudinal and motivational part of the students.

Keywords: Reading levels, critical reading, didactic unit, meaningful learning.

\section{Resumo}

A pesquisa surge da necessidade de fortalecer a leitura crítica nos alunos décimo primeiro grau, porque depois de analisar os resultados dos testes identificados Sabendo que os níveis de leitura dos alunos não eram adequadas. Para fazer isso, várias investigações sobre os problemas mencionados, que por sua vez serviram como estado da arte para o processo de pesquisa, e estes trabalhos grau feito em mestrado e doutorado a nível nacional e internacional consultados, respectivamente, é importante notar que Depois de realizar uma pesquisa abrangente no nível regional, nenhuma pesquisa foi encontrada que contribuiu significativamente para o processo.

O conjunto principal objetivo foi o de promover a leitura crítica dos alunos, com base na determinação do nível de leitura estudantes (literais, inferencial e críticos) através de um teste de diagnóstico. Mais tarde, foi projetada e implementada uma unidade de ensino como uma estratégia de ensino inovador, que através de suas atividades focadas na aprendizagem significativa permitiria o fortalecimento da leitura crítica. Acima, ele fez parte da metodologia da pesquisa-ação, com abordagem qualitativa uma vez que todo o processo é auto-reflexivo e inquirir sobre oportunidades para melhorar tanto o professor e aluno; Também foi aplicado em um contexto pedagógico natural, jogando dados descritivos e revelando, necessidades sociais, culturais educacional, instrutivo, entre outros.

Então, foi possível determinar o nível de leitura de cada unidade de estudante e de ensino, desenvolvido como uma estratégia de ensino foi plenamente aplicado, e ficou satisfeito por eles. Finalmente, o progresso de 
cada um dos alunos nos três níveis de leitura foi avaliado pela aplicação de um teste final.

Finalmente, foi determinado que deve ser reforçada a leitura literal, com tudo o que isso implica, que os outros níveis são devidamente desenvolvido, tendo em conta que esse reforço também deve ser focada na parte de atitude e motivação dos alunos.

Palavras-chave: níveis de leitura, leitura crítica, unidade didática, aprendizagem significativa.

\section{Introducción}

Teniendo en cuenta la necesidad que motivó el trabajo investigativo, aparecen conceptos que son necesarios tenerlos claros para poder generar y comprender el andamiaje en el que se sustenta la investigación. De esta manera, Solé (1992) entiende el acto de leer como comprender e interpretar textos escritos de diverso tipo con diferentes intenciones y objetivos, que contribuye de manera decisiva a la autonomía de las personas, en la medida en que la lectura es un instrumento necesario para manejarse con ciertas garantías en una sociedad letrada (p. 9). Por otra parte, Cassany (2006) dice:

leer implica decodificar las palabras del texto, exige que el lector aporte conocimientos previos, obliga a inferir todo lo que no se dice. Pero la comprensión proviene de la comunidad de hablantes; el significado nace de la cultura que comparten el autor y el lector. (p. 13)

Teniendo en cuenta lo expuesto por los autores es evidente que leer es un proceso complejo que tiene como finalidad relacionarse con el otro, comprender intenciones y contextos con el objetivo de ser aceptado. Sin embargo, el no desarrollar adecuadamente los niveles de lectura como los plantea Cassany (2006): Lectura de las líneas, que se basa en comprender el significado literal (la suma del significado semántico de todas sus palabras); Lectura entre líneas, que es lo que se deduce de las palabras, las inferencias, las presuposiciones, la ironía, los dobles sentidos, etc.; y Lectura detrás de las líneas, donde se encuentra la ideología, el punto de vista, la intención y la argumentación dada por el autor; conllevará a un bajo desempeño en cualquier labor académica, social, cultura y democrática.

Otro punto de vista lo aporta David Klooster (2001) citado por Cassany (2003) cuando ofrece un listado de habilidades que un buen lector debe poseer para llegar a un pensamiento crítico, estos son: cada sujeto debe construir su propio conocimiento desde la individualidad; se requiere conocimientos o información 
adicional a la leída; el proceso de lectura debe iniciar con preguntas o problemas que interesan al sujeto y que debe resolver; al leer se debe reflexionar y buscar argumentaciones razonadas ( $\mathrm{p}$. 117).

En conclusión, Cassany y Castellá (2010) proponen una serie de destrezas que un lector crítico debe poseer para que emita juicios, argumentos $\mathrm{u}$ opiniones con responsabilidad, con tolerancia y tenga una fluida interacción en el intercambio de ideas. Según estos autores, lo anterior se puedo conseguir conociendo el léxico y las similitudes que se encuentran en el texto; conociendo las expresiones del texto, darles el verdadero significado, distinguiendo entre lo imaginario y lo real planteado en el texto; identificando el punto de vista del autor respecto al texto; distinguiendo la ironía, sarcasmo y doble sentido del texto; distinguiendo la diversidad de voces del texto (citas, referencias...); valorando la importancia de las diferentes voces dentro del texto; identificando el género discursivo en el que se inscribe el texto; identificando los argumentos, el propósito o la intención del autor; exponiendo puntos de vista alternativos; determinando el contexto del texto; analizando discursos previos o posteriores al actual y conociendo al autor del texto (p. 365).

Teniendo en cuenta lo postulado anteriormente se crearon las actividades en cada una de las sesiones de la unidad didáctica como estrategia innovadora para el fortalecimiento de la lectura crítica en los estudiantes del grado undécimo de la Institución Educativa Alonso Carvajal Peralta.

Los diferentes motivos que llevaron a la creación de la unidad didáctica como propuesta pedagógica para fortalecer la lectura crítica en los estudiantes de undécimo grado surgen, en primera medida, por los resultados de las pruebas saber $9^{\circ}$ - 2016, los cuales sugieren que los estudiantes se encuentran en un nivel medio en la escala nacional en cuanto a lectura crítica. Por otra parte, es evidente en las aulas de clase los bajos niveles de lectura comprensiva y reflexiva, además de los pocos argumentos o pobres puntos de vista acerca de un tema de interés.

Ahondemos un poco más, la mayoría de los jóvenes que cursan el bachillerato y más preocupante aún aquellos próximos a graduarse, no realizan una lectura consiente, rica en perspectivas, y no identifican adecuadamente los elementos literales e inferenciales de los textos, ya sean escritos, orales, gráficos, gestuales, entre otros. En consecuencia, no alcanzan una lectura crítica, teniendo en cuenta que cada nivel de lectura es requisito del siguiente, lo que conduce a que su lectura está sujeta sólo a la decodificación y esto no le permite ver más allá del texto que lee. 
En el mismo orden de ideas, se consideró relevante la elaboración y posterior desarrollo de la presente propuesta pedagógica porque pretende, a través de las sesiones que conforman la unidad didáctica, contribuir a la reflexión de cada estudiante sobre el contexto en el que se desenvuelve, ya sea social, educativo, cultural, democrático y próximamente profesional. Dicho con otras palabras, cada estudiante debe tener la capacidad de comparar, analizar, sintetizar y elaborar sus propios puntos de vista con lo que vea, viva, lea y sienta a su alrededor.

\section{Metodología}

En efecto, la unidad didáctica fue pensada desde la perspectiva de una estructura de trabajo que se plantea unos objetivos claros, medibles y alcanzables con un cúmulo de actividades secuenciales y dispuestas para adquirir conocimientos ó habilidades especificas. Dicho esto, la unidad didáctica se elabora con base en los aprendizajes que los estudiantes fallan más, estos son: "no relacionan, identifican ni deducen información para construir el sentido global del texto y no relacionan textos ni moviliza saberes previos para ampliar referentes y contenidos ideológicos" (ICFES 2017).

Teniendo en cuenta lo anterior, se diseñaron 8 sesiones donde el mapa mental, la historieta, el aviso publicitario y la infografía, el texto expositivo, el texto argumentativo y el juego de roles -traídos del currículo escolar- jugaron un papel muy importante en el desarrollo de la competencia de lectura crítica. Con respecto al tiempo de aplicación de la unidad didáctica de lectura crítica, se desarrolló en 11 semanas, que se distribuyeron de la siguiente manera: prueba diagnóstica, sesiones 1 a la 4; prueba intermedia, sesiones 5 a la 8; y prueba final. Aunque estos tiempos fueron un estimado, ya que es muy común en las instituciones educativas públicas que el calendario académico se vea afectado por diferentes factores tanto internos como externos.

Otro aspecto relevante que es necesario aclarar es que las diferentes temáticas que propone la unidad didáctica se relacionan entre sí de manera secuencial, ya que a medida que se introducen los nuevos tópicos se plasman actividades que buscan retroalimentar las ya realizadas y evaluadas. De esta manera los talleres de cada sesión que conforman la unidad didáctica se retroalimentan y generan cohesión.

Para entender un poco más la estructura y metodología de la unidad didáctica, cabe aclarar que se divide en dos partes. La primera hace referencia al aprendizaje: relaciona, identifica y deduce información para construir el sentido global del texto, donde se desarrollaron concernientes a textos discontinuos como el mapa 
mental, la historieta, el aviso publicitario y la infografía. La segunda parte tiene que ver con el aprendizaje: relaciona textos y moviliza saberes previos para ampliar referentes y contenidos ideológicos. En este último se trabajó a partir de textos continuos como los son el expositivo y el argumentativo.

Lo dicho hasta aquí supone un fundamento pedagógico que debe contener la unidad didáctica y partiendo del hecho que en la Institución Educativa Alonso Carvajal Peralta se tiene al constructivismo como modelo pedagógico, que a grandes rasgos se puede sintetizar al decir que cada estudiante es el responsable de construir su conocimiento a partir de sus perspectivas sociales y experiencias de vida, reestructurando los presaberes con los nuevos saberes adquiridos durante el proceso de aprendizaje. En este orden de ideas, se considera al docente como un orientador del estudiante en la adquisición y construcción del conocimiento mediante la actividad y la experiencia, donde juega un papel importante el currículo por procesos que debe estar abierto y permeable a la influencia del contexto en el cual está inmersa la institución educativa.

\section{Conclusiones}

Comencemos por decir que se logró determinar el nivel de lectura (literal, inferencial y crítico) de los estudiantes del grado undécimo, por medio de una prueba diagnóstica elaborada con preguntas de exámenes saber 11 anteriores, liberadas por el ICFES.

Teniendo en cuenta los resultados anteriores, se diseña e implementa una unidad didáctica como estrategia pedagógica innovadora que permitió en gran parte de los estudiantes el fortalecimiento de los tres niveles de lectura. Para diseñar la unidad didáctica se tuvo en cuenta el P.E.I. de la Institución Educativa, el cual tiene como fundamento el modelo pedagógico del constructivismo y por ende el aprendizaje significativo, además los planteamientos de diferentes teóricos que son una autoridad en el tema. Teniendo claro esto, todas las actividades giraron en torno a este modelo pedagógico y postulados teóricos.

Por otra parte, durante la implementación de la unidad didáctica como estrategia pedagógica innovadora se presentaron modificaciones tanto de forma como de fondo, porque la muestra poblacional se detectó muy heterogénea en cuanto a hábitos de estudio, estrategias de lectura, gusto por la lectura, capacidad de concentración y resistencia a dejar de lado el modelo tradicional de aprendizaje.

Por otra parte, durante todo el proceso de ejecución de la unidad didáctica se evalúo al aplicar una prueba diagnóstica, una prueba intermedia, una prueba final y al concluir cada una de las ocho 
sesiones se realizó una heteroevaluación, coevaluación y una autoevaluación. Esto indica que todo el proceso estuvo en constante valoración y así se facilitó detectar el avance en el fortalecimiento de la lectura crítica.

Teniendo en cuenta las conclusiones mencionadas anteriormente, surge una nueva y quizás la más importante de todas, sin lugar a dudas: el rol del docente es fundamental en el proceso de enseñanza aprendizaje $y$ este proceso tendrá éxito solo cuando se rompa el paradigma del modelo pedagógico tradicional, pues sin darse cuenta se encasilla a los estudiantes y con el pasar del tiempo es complejo modificar conductas que entorpecen la adquisición, desarrollo y fortalecimiento del pensamiento crítico en las aulas de clase.

Para terminar, es indispensable traer al aula o fuera de ella, actividades que generen interés y motivación hacia la lectura, desarrollo de la imaginación, debates de opiniones, realizar diversas consultas $y$ posturas sobre el mismo tema, usando herramientas tecnológicas y permitirle al estudiante que sea el protagonista de su proceso de aprendizaje.

\section{Referencias}

Cassany D. (2003). Aproximaciones a la lectura crítica: teoría, ejemplos y reflexiones. Tarbiya (32), p. 113-132. Recuperado de https://revistas.uam.es/tarbiy

a/article/viewFile/7275/7623

Cassany D. (2006). Tras las líneas sobre la lectura contemporánea. Barcelona, España: Ed. Anagrama. Recuperado de https://bit.ly/2xRvDpU

Cassany D y Castellá J. (2010). Aproximación a la literacidad crítica. Recuperado de: https://bit.ly/2FNMQz4

Solé, I. (1992). Estrategias de lectura. Barcelona, España: Editorial Graò. Recuperado de https://bit.ly/2nn3pxs 


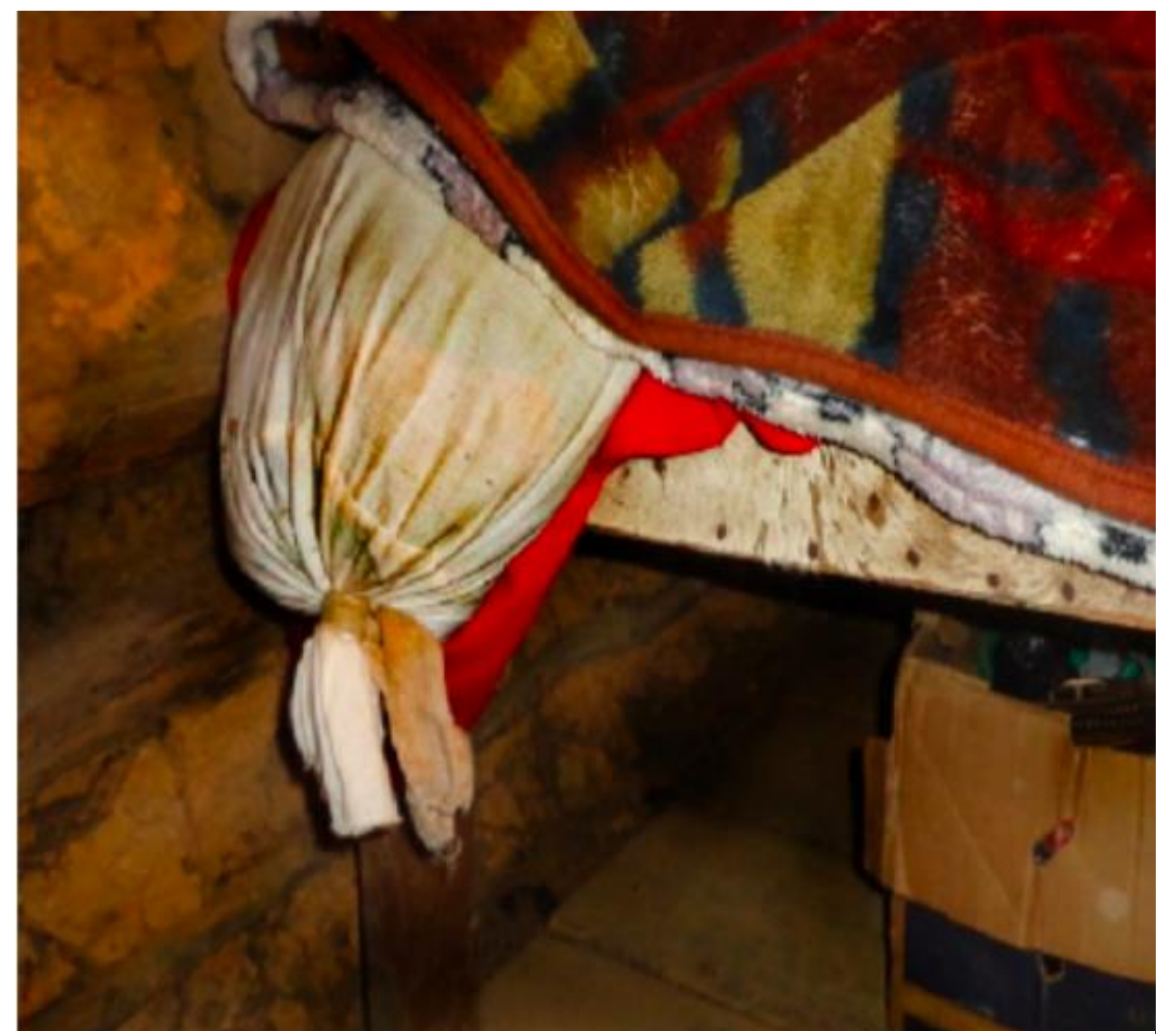

Número de la obra: 3

Título: "sobre camas"

Autor: Yenny Banesa Bonilla

Lugar: Zona rural municipio de Baraya

Finca: La Lira

Fecha de captura: Febrero 18 de 2014

Técnica: Fotografía digital

Fuente: Bonilla, Y. B. (2014). Sobre camas. Una mirada estética popular del municipio de Baraya. Tesis de pregrado. Universidad Surcolombiana. Neiva, 2014. p. 72. 